T. Ohsawa

Nagoya Math. J.

Vol. 142 (1996), 1-4

\title{
A NOTE ON THE VARIATION OF RIEMANN SURFACES
}

\author{
TAKEO OHSAWA
}

1. Let $X$ be any Riemann surface. By Koebe's uniformization theorem we know that the universal covering space of $X$ is conformally equivalent to either Riemann sphere, complex plane, or the unit disc in the complex plane. If $X$ is allowed to vary with parameters we may inquire the parameter dependence of the corresponding family of the universal covering spaces.

2. This question was essentially answered by the theory of simultaneous uniformization due to L. Bers [B]. According to this theory, for any complex analytic family of compact Riemann surfaces of genus $\geq 2$ over a simply connected complex manifold $M$, the universal covering of the total space of the family is biholomorphically equivalent to a locally pseudoconvex domain in $M \times \boldsymbol{C}$ on which the covering transformation group acts as a complex analytic family of quasi-Fuchsian groups. This picture was extended by Earle and Fowler $[\mathrm{E}-\mathrm{F}]$ to a certain class of variations of open Riemann surfaces.

3. On the other hand, H. Yamaguchi $[Y]$ pursued the variation of open Riemann surfaces of class $O_{A D}$ along the ideas initiated by K. Oka and T. Nishino, and succeeded in realizing the simultaneous Schottky uniformization of compact Riemann surfaces of genus $\geq 2$ parametrized over the unit disc, completely independently of the method of Bers.

4. Therefore one may ask for a generalization of Yamaguchi's theory to open Riemann surfaces, or as an ultimate purpose for a simultaneous uniformization theory for an arbitrary Kleinian group.

5. Since few things seem to be known about such a generalized uniformization problem, we would like to proceed first by establishing a general result that holds for any covering space of a complex analytic family of compact Riemann

Received January 18, 1995. 
surfaces over the unit disc.

6. In order to state the result, let $(\mathscr{X}, U, \pi)$ be a triple consisting of a (connected) complex manifold $\mathscr{X}$ of dimension 2, the unit disc $U \subset \boldsymbol{C}$ and a proper surjective holomorphic map $\pi: \mathscr{X} \rightarrow U$ of maximal rank. What we want to prove is:

THEOREM 1. Every covering space of $\mathscr{X}$ is holomorphically convex.

7. This will turn out to be a direct consequence of a more general result, which can be formulated as follows.

Theorem 2. Let $E$ be a complex manifold of dimension 2. Suppose that there exists a surjective holomorphic map $\varpi: E \rightarrow U$ satisfying the following condition.

(*) There exists a family of holomorphic sections $s_{y}: U \rightarrow E$ with parameter $y$ running through $E_{0}:=\varpi^{-1}(0)$ such that $E$ is the disjoint union of the subsets $\left\{s_{y}(U)\right\}_{y \in E_{0}}$.

Then $E$ is holomorphically convex.

8. In fact, in virtue of the lifting theorem of Earle-Kra-Krushkal [E-K-K], $\mathscr{X}$ satisfies the condition of Theorem 2 , and a fortiori so does every covering space of $\mathscr{X}$. So we are going to prove Theorem 2 in the next paragraph.

9. There is nothing to prove if $\varpi$ is proper. Hence let us assume that $E_{0}$ is noncompact. Note that $E_{t}=\varpi^{-1}(t)$ are then all noncompact and homeomorphic to $E_{0}$ by the condition (*). Since $E$ is connected and $U$ is simply connected, $E_{t}$ are connected, too. Therefore $E_{t}$ are all Stein manifolds by Behnke-Stein's theorem (cf. [B-S]) so that, in particular, $E_{0}$ admits a strictly subharmonic exhaustion function $\varphi: E_{0} \rightarrow[0, \infty)$ of class $C^{\infty}$. We set $E_{0}^{c}=\{p \mid \varphi(p)<c\}$. Then the set

$$
E^{c}:=\bigcup_{y \in E_{0}^{c}} s_{y}(U)
$$

is an open subset of $E$, since the map

$$
\begin{aligned}
s: U \times E_{0} & \rightarrow E \\
\Psi & \Psi \\
(t, y) & \mapsto s_{y}(t)
\end{aligned}
$$

is jointly continuous in $(t, y)$ (cf. [B-R]). Clearly $E^{\mathrm{c}}$ is locally pseudoconvex in $E$. We assert that there exist a neighbourhood $V$ of the closure of $E^{c}$ in $E$ and a $C^{\infty}$ 
strictly plurisubharmonic function $\phi$ defined on $V$. To see this, and to specify such a function, let $\left\{U_{i}\right\}_{i \in \mathbf{N}}$ be a locally finite covering of $U$ such that one has neighbourhoods

$$
V_{i} \supset \overline{E^{c}} \cap \varpi^{-1}\left(U_{i}\right)
$$

and $C^{\infty}$ strictly plurisubharmonic functions $\phi_{i}: V_{i} \rightarrow[0, \infty)$. This procedure is possible because Stein subvarieties always admit Stein neighbourhood systems (cf. $[\mathrm{S}]$ ). Let $\left\{\rho_{i}\right\}_{i \in \mathbf{N}}$ be a (nonnegative) $C^{\infty}$ partition of unity subordinate to $\left\{U_{i}\right\}_{i \in \mathbf{N}}$. Then it is clear that the function

$$
\phi(p):=\sum_{i \in \mathbf{N}} \rho_{i}(p) \phi_{i}(p)+\lambda\left(|\varpi(p)|^{2}\right)
$$

is strictly plurisubharmonic on a neighbourhood of $\overline{E^{c}}$ if $\lambda$ is a $C^{\infty}$ convex increasing function of sufficiently rapid growth. Let $d s^{2}$ be any Kähler metric on $V$, for instance one may take the complex Hessian of as $d s^{2}$, and let $\delta_{c}(p)$ denote the geodesic distance from $p$ to the boundary of $E^{c}$ measured with respect to $d s^{2}$. Then the Levi form of $-\log \delta_{c}$ is locally bounded from below as a $(1,1)$-current (cf. $[\mathrm{E}]$ ), so that one can find a convex increasing function $\eta$ such that the function $-\log \delta_{c}+\eta \circ \phi$ is exhaustive and has everywhere positive Levi form as a $(1,1)$-current. This implies that $E^{\mathrm{c}}$ is a Stein manifold (cf. the approximation theorem of Richberg $[\mathrm{R}]$ and Grauert's solution to the Levi problem $[G]$ ). Since $E$ is an increasing union of the continuous family of Stein open subsets $\left\{E^{c}\right\}_{c \in \mathbf{R}^{\prime}}$ we conclude from [D-G, Satz 18] that $E$ is also a Stein manifold.

\section{REFERENCES}

[B-S] Behnke, H. and Stein, K., Entwicklung analytischer Funktionen auf Riemannschen Flächen, Math. Ann., 120 (1948), 430-461.

[B-R] Bers, L. and Royden, H., Holomorphic families of injections, Acta Math., 157 (1986), 259-289.

[D] Demailly, J.-P., Cohomology of $q$-convex spaces in top degrees, Math. Z., 204 (1990), 283-295.

[D-G] Docquier, F. and Grauert, H., Levisches Problem und Rungescher Satz für Teilgebiete Steinscher Mannigfaltigkeiten, Math. Ann., 140 (1960), 94-123.

[E-F] Earle, C. J. and Fowler, S., Holomorphic families of open Riemann surfaces, Math. Ann., 270 (1985), 249-273.

[E-K-K] Earle, C. J., Kra, I. and Krushkal', S. L., Holomorphic motions and Teichmüller spaces, Trans. AMS., 343 (1994), 927-948.

[E] Elencwajg, G., Pseudo-convexité locale dans les variétés Kähleriennes, Ann. Inst. Fourier, 25 (1975), 295-314.

[G] Grauert, H., On Levi's problem and the imbedding of real-analytic manifolds, Ann. Math., 68 (1958), 460-472. 
[O] Ohsawa, T., Completeness of noncompact analytic spaces, Publ. RIMS, 20 (1984), 683-692.

[R] Richberg, R., Stetige streng pseudokonvexe Funktionen, Math. Ann., 175 (1968), 251-286.

[S] Siu, Y. T., Every Stein subvariety has a Stein neighbourhood, Invent. Math., 38 (1976), 89-100.

[Y] Yamaguchi, H., Variations de surfaces de Riemann, C. R. Acad. Sc., 286 (1978), $1121-1124$

Graduate School of Polymathematics

Nagoya University

Chikusa-ku, Nagoya 464-01

Japan 